\title{
AN APPLICATION OF MITTAG-LEFFLER TYPE POISSON DISTRIBUTION SERIES ON STARLIKE AND CONVEX FUNCTIONS
}

\author{
SAURABH PORWAL ${ }^{1, *}$, DIVYA BAJPAI ${ }^{2}$ \\ ${ }^{1}$ Lecturer Mathematics, Sri Radhey Lal Arya Inter College, Ehan, Hathras-204101, (U.P.), India \\ ${ }^{2}$ Department of Mathematics, UIET, CSJM University, Kanpur-208024, (U.P.), India \\ Corresponding author: saurabhjcb@rediffmail.com \\ Received May 26, 2019
}

AвSTRACт. In the present paper authors obtain some sufficient conditions on Mittag-Leffler type Poisson distribution series on certain classes of starlike and convex functions.

2010 Mathematics Subject Classification. 30C45.

Key words and phrases. analytic; starlike; convex; Mittag-Leffler type Poisson distribution.

\section{InTRODUCTION}

Let $A$ denote the class of functions $f$ of the form

$$
f(z)=z+\sum_{n=2}^{\infty} a_{n} z^{n}
$$

which are analytic in the open unit disk $U=\{z: z \in C$ and $|z|<1\}$ and satisfy the normalization condition $f(0)=f^{\prime}(0)-1=0$. Further, we denote by $S$ the subclass of $A$ consisting of functions of the form (1.1) which are also univalent in $U$.

Denote by $T$ the subclass of $A$ consisting of functions whose non zero coefficients from second on, is given by

$$
f(z)=z-\sum_{n=2}^{\infty}\left|a_{n}\right| z^{n} .
$$

A function $f$ of the form (1.1) is said to be starlike of order $\alpha$ if it satisfies the following condition

$$
\Re\left\{\frac{z f^{\prime}(z)}{f(z)}\right\}>\alpha, z \in U, 0 \leq \alpha<1,
$$

and is said to be convex of order $\alpha$ if it satisfies the following condition

$$
\Re\left\{1+\frac{z f^{\prime \prime}(z)}{f^{\prime}(z)}\right\}>\alpha, z \in U, 0 \leq \alpha<1 .
$$

The classes of all starlike and convex functions of order $\alpha$ are denoted by $S^{*}(\alpha)$ and $K(\alpha)$ respectively, studied by Robertson [13] and Silverman [14].

DOI: $10.28924 / \mathrm{APJM} / 7-1$ 
The application of Hypergeometric function, Generalized Hypergeometric function, Wright function, Generalized Bessel function and Poisson distribution series on various subclasses of analytic and harmonic univalent functions are play an important and significant role in Geometric Function Theory. It establishes a connection between Special functions, Statistics and Complex analysis. In this direction the works of ( [1], [4], [5], [7]- [11] and [15]) give a significant contribution in the field. Very recently, Porwal and Dixit [12] investigated MittagLeffler type Poisson distribution which is a generalization of Poisson distribution. Therefore it is natural to ask whether Poisson distribution series [6] can be extended to Mittag-Leffler type Poisson distribution series.

Motivating with this question and the work of ( [3], [6], [11]) we give an application of Mittag-Leffler type Poisson distribution series on univalent functions. The Mittag-Leffler type Poisson distribution is given by

$$
P(m, \alpha, \beta, k)=\frac{m^{k}}{E_{\alpha, \beta}(m) \Gamma(\alpha k+\beta)}, k=0,1,2,3 \ldots
$$

where $m>0, \alpha>0, \beta>0$ and $E_{\alpha, \beta}(m)=\sum_{k=0}^{\infty} \frac{m^{k}}{\Gamma(\alpha k+\beta)}$.

For detailed study see [12].

Now we introduce Mittag-Leffler type Poisson distribution series in the following way-

$$
K(\alpha, \beta, m, z)=z+\sum_{n=2}^{\infty} \frac{m^{n-1}}{\Gamma(\alpha(n-1)+\beta)} \frac{1}{E_{\alpha, \beta}(m)} z^{n} .
$$

If $\alpha=1, \beta=1$ then we obtain Poisson distribution series [6],

$$
K(1,1, m, z)=z+\sum_{n=2}^{\infty} \frac{m^{n-1}}{(n-1) !} \frac{1}{e^{m}} z^{n} .
$$

The application of Poisson distribution series on Geometric Function Theory is a recent topic of study. Note worthy contribution in this field are ( [4], [8], [10]).

The convolution (or Hadamard product) of two power series of the form

$$
f(z)=\sum_{n=0}^{\infty} a_{n} z^{n} \text { and } g(z)=\sum_{n=0}^{\infty} b_{n} z^{n}
$$

is defined as the power series

$$
(f * g)(z)=\sum_{n=0}^{\infty} a_{n} b_{n} z^{n}
$$

Now we consider the linear operator $I(\alpha, \beta, m): A \rightarrow A$ defined by

$$
\begin{aligned}
I(\alpha, \beta, m) f(z) & =k(\alpha, \beta, m, z) * f(z) \\
& =z+\sum_{n=2}^{\infty} \frac{m^{n-1}}{\Gamma(\alpha(n-1)+\beta)} \frac{1}{E_{\alpha, \beta}(m)} a_{n} z^{n} .
\end{aligned}
$$

To prove our main results we shall require the following lemmas. Lemma 1.1. ( [2]) If $f \in R^{\tau}(A, B)$ is of the form (1.1) then

$$
\left|a_{n}\right| \leq \frac{(A-B)|\tau|}{n},(n \in N /\{1\})
$$

The bounds given in (1.8) is sharp. 
Lemma 1.2. ( [14]) Let $f \in A$ be of the form (1.1) then $f \in S^{*}(\delta)$, if

$$
\sum_{n=2}^{\infty}(n-\delta)\left|a_{n}\right| \leq 1-\delta
$$

Lemma 1.3.( [14]) Let $f \in A$ be of the form (1.1) and satisfy the conditon

$$
\sum_{n=2}^{\infty} n(n-\delta)\left|a_{n}\right| \leq 1-\delta
$$

then $f \in K(\delta)$.

In this paper, motivated with the above mentioned work and work of Kumar [3], we obtain some sufficient conditions for the function $K(\alpha, \beta, m, z)$ belonging to the classes $S^{*}(\delta)$ and $K(\delta)$ and connections of these subclasses with $R^{\tau}(A, B)$.

\section{Main Results}

Theorem 2.1. If $\alpha, m>0, \beta>1$, then $K(\alpha, \beta, m, z) \in S^{*}(\delta)$, if

$$
\frac{1}{\alpha} \frac{1}{E_{\alpha, \beta}(m)}\left[\left(E_{\alpha, \beta-1}(m)-\frac{1}{\Gamma \beta-1}\right)+\{\alpha(1-\delta)-(\beta-1)\}\left(E_{\alpha, \beta}(m)-\frac{1}{\Gamma \beta}\right)\right] \leq 1-\delta .
$$

Proof. To prove $K(\alpha, \beta, m, z) \in S^{*}(\delta)$, from Lemma 1.2, it suffices to prove that

$$
\sum_{n=2}^{\infty}(n-\delta) \frac{1}{E_{\alpha, \beta}(m)} \frac{m^{n-1}}{\Gamma(\alpha(n-1)+\beta)} \leq 1-\delta .
$$

$$
\text { Now } \begin{aligned}
\frac{1}{E_{\alpha, \beta}(m)} \sum_{n=2}^{\infty}(n-\delta) \frac{m^{n-1}}{\Gamma(\alpha(n-1)+\beta)} \\
\quad=\frac{1}{E_{\alpha, \beta}(m)} \sum_{n=2}^{\infty} \frac{(n-\delta)}{(\alpha(n-1)+\beta-1)} \frac{m^{n-1}}{\Gamma(\alpha(n-1)+\beta-1)} \\
=\frac{1}{E_{\alpha, \beta}(m)} \sum_{n=2}^{\infty} \frac{1}{\alpha} \frac{(n-\delta)}{\left[\left[(n-1)+\left(\frac{\beta-1}{\alpha}\right)\right]\right.} \frac{m^{n-1}}{\Gamma(\alpha(n-1)+\beta-1)} \\
=\frac{1}{E_{\alpha, \beta}(m)} \frac{1}{\alpha} \sum_{n=2}^{\infty} \frac{\left[\left(n-1+1+\left(\frac{\beta-1}{\alpha}\right)-\left(\frac{\beta-1}{\alpha}\right)-\delta\right]\right.}{\left.(n-1)+\left(\frac{\beta-1}{\alpha}\right)\right) \Gamma(\alpha(n-1)+\beta-1)} m^{n-1} \\
=\frac{1}{E_{\alpha, \beta}(m)} \frac{1}{\alpha} \sum_{n=2}^{\infty} \frac{\left\{(n-1)+\left(\frac{\beta-1}{\alpha}\right)-\left(\frac{\beta-1}{\alpha}\right)+(1-\delta)\right\} m^{n-1}}{\left\{(n-1)+\left(\frac{\beta-1}{\alpha}\right) \Gamma(\alpha(n-1)+\beta-1)\right\}} \\
=\frac{1}{E_{\alpha, \beta}(m)} \frac{1}{\alpha} \sum_{n=2}^{\infty}\left[\frac{m^{n-1}}{\Gamma(\alpha(n-1)+\beta-1)}-\frac{(\beta-1) m^{n-1}}{\Gamma(\alpha(n-1)+\beta)}+\frac{\alpha(1-\delta) m^{n-1}}{\Gamma(\alpha(n-1)+\beta)}\right] \\
=\frac{1}{\alpha E_{\alpha, \beta}(m)}\left[\left(E_{\alpha, \beta-1}(m)-\frac{1}{\Gamma(\beta-1)}\right)+\{\alpha(1-\delta)-(\beta-1)\}\left(E_{\alpha, \beta}(m)-\frac{1}{\Gamma(\beta)}\right)\right] .
\end{aligned}
$$

But, this last expression is bounded above by $1-\delta$, if (2.1) holds.

This complete the proof of Theorem 2.1.

Theorem 2.2. If $\alpha, m>0, \beta>2$, then $K(\alpha, \beta, m, z) \in K(\delta)$ if

$$
\frac{1}{\alpha^{2} E_{\alpha, \beta}(m)}\left[\begin{array}{l}
E_{\alpha, \beta-2}(m)-\frac{1}{\Gamma(\beta-2)}+(3-2 \beta-\alpha-\alpha \delta)\left(E_{\alpha, \beta-1}(m)-\frac{1}{\Gamma(\beta-1)}\right) \\
+\left\{(1-\beta+\alpha)^{2}-\delta \alpha(1-\beta+\alpha)\right\}\left(E_{\alpha, \beta}(m)-\frac{1}{\Gamma(\beta)}\right)
\end{array}\right] \leq 1-\delta .
$$


Proof. To prove $K(\alpha, \beta, m, z) \in K(\delta)$, from Lemma 1.3, it is sufficient to prove that

$$
\sum_{n=2}^{\infty} n(n-\delta) \frac{1}{E_{\alpha, \beta}(m)} \frac{m^{n-1}}{\Gamma(\alpha(n-1)+\beta)} \leq 1-\delta
$$

Now

$$
\begin{aligned}
& \sum_{n=2}^{\infty} n(n-\delta) \frac{1}{E_{\alpha, \beta}(m)} \frac{m^{n-1}}{\Gamma(\alpha(n-1)+\beta)} \\
& =\sum_{n=2}^{\infty}\left(n^{2}-n \delta\right) \frac{1}{E_{\alpha, \beta}(m)} \frac{m^{n-1}}{\Gamma(\alpha(n-1)+\beta)} \\
& =\frac{1}{E_{\alpha, \beta}(m)}\left[\sum_{n=2}^{\infty} \frac{\left(n^{2}-n \delta\right)}{\{\alpha(n-1)+\beta-1\}\{\alpha(n-1)+\beta-2\}} \frac{m^{n-1}}{\Gamma(\alpha(n-1)+\beta-2)}\right] \\
& =\frac{1}{E_{\alpha, \beta}(m)}\left[\sum_{n=2}^{\infty} \frac{1}{\alpha^{2}} \frac{\left(n^{2}-n \delta\right)}{\left\{(n-1)+\left(\frac{\beta-1}{\alpha}\right)\right\}\left\{\left(n-1+\frac{(\beta-2)}{\alpha}\right\}\right.} \frac{m^{n-1}}{\Gamma(\alpha(n-2)+\beta-2)}\right] \\
& =\frac{1}{E_{\alpha, \beta}(m)} \sum_{n=2}^{\infty}\left[\left\{(n-1)+\left(\frac{\beta-1}{\alpha}\right)\right\}\left\{(n-1)+\left(\frac{\beta-2}{\alpha}\right)\right\}+\left(\frac{3-2 \beta+2 \alpha-\alpha \delta}{\alpha}\right)\right. \\
& \left.\times\left\{(n-1)+\left(\frac{\beta-1}{\alpha}\right)\right\}+\left(\frac{1-\beta+\alpha}{\alpha}\right)^{2}-\frac{\delta(1-\beta+\alpha)}{\alpha}\right] \frac{m^{n-1}}{\Gamma(\alpha(n-1)+\beta)} \\
& =\frac{1}{E_{\alpha, \beta}(m)} \sum_{n=2}^{\infty}\left[\frac{1}{\alpha^{2}}\{\alpha(n-1)+\beta-1\}\{\alpha(n-1)+\beta-2\}+\left(\frac{3-2 \beta+2 \alpha-\alpha \delta}{\alpha}\right)\right. \\
& \left.\times\left(\frac{\alpha(n-1)+\beta-1}{\alpha}\right)+\left(\frac{1-\beta+\alpha}{\alpha}\right)^{2}-\frac{\delta(1-\beta+\alpha)}{\alpha}\right] \frac{m^{n-1}}{\Gamma(\alpha(n-1)+\beta)} \\
& =\frac{1}{E_{\alpha, \beta}(m)}\left[\frac{1}{\alpha^{2}} \sum_{n=2}^{\infty} \frac{m^{n-1}}{\Gamma(\alpha(n-1)+\beta-2)}+\left(\frac{3-2 \beta+2 \alpha-\alpha \delta}{\alpha^{2}}\right)\right. \\
& \left.\times \sum_{n=2}^{\infty} \frac{m^{n-1}}{\Gamma(\alpha(n-1)+\beta-1)}+\left\{\left(\frac{1-\beta+\alpha}{\alpha}\right)^{2}-\frac{\delta(1-\beta+\alpha)}{\alpha}\right\} \sum_{n=2}^{\infty} \frac{m^{n-1}}{\Gamma(\alpha(n-1)+\beta)}\right] \\
& =\frac{1}{E_{\alpha, \beta}(m)} \frac{1}{\alpha^{2}}\left[\begin{array}{c}
\left(E_{\alpha, \beta-2}(m)-\frac{1}{\Gamma(\beta-2)}\right)+(3-2 \beta+2 \alpha-\alpha \delta)\left(E_{\alpha, \beta-1}(m)-\frac{1}{\Gamma(\beta-1)}\right) \\
+\left\{(1-\beta+\alpha)(1-\beta+\alpha-\alpha \delta)\left(E_{\alpha, \beta}(m)-\frac{1}{\Gamma(\beta)}\right)\right\}
\end{array}\right] \\
& \leq 1-\delta
\end{aligned}
$$

This completes the proof of Theorem 2.2.

Theorem 2.3. If $\alpha, \beta, m>0, f \in R^{\tau}(A, B)$ and the inequality

$$
\frac{(A-B)|\tau|}{E_{\alpha, \beta}(m)}\left[\left\{E_{\alpha, \beta}(m)-\frac{1}{\Gamma(\beta)}\right\}-\delta\left\{\int_{0}^{m} \frac{E_{\alpha, \beta}(t)}{t} d t-\frac{1}{\Gamma(\beta)}\right\}\right] \leq 1-\delta,
$$

is satisfied then $I(\alpha, \beta, m) f(z) \in S^{*}(\delta)$. 
Proof. The function $I(\alpha, \beta, m) f(z)$ is given by (1.7).

To prove $I(\alpha, \beta, m) f(z) \in S^{*}(\delta)$ we have to prove that

$$
\sum_{n=2}^{\infty}(n-\delta) \frac{m^{n-1}}{\Gamma(\alpha(n-1)+\beta)} \frac{1}{E_{\alpha, \beta}(m)}\left|a_{n}\right| \leq 1-\delta .
$$

Since $f(z) \in R^{\tau}(A, B)$, from Lemma 1.1 we have

$$
\left|a_{n}\right| \leq \frac{(A-B)|\tau|}{n} .
$$

Now

$$
\begin{gathered}
\sum_{n=2}^{\infty}(n-\delta) \frac{m^{n-1}}{\Gamma(\alpha(n-1)+\beta)} \frac{1}{E_{\alpha, \beta}(m)}\left|a_{n}\right| \\
\leq \frac{(A-B)|\tau|}{E_{\alpha, \beta}(m)} \sum_{n=2}^{\infty} \frac{(n-\delta)}{n \Gamma(\alpha(n-1)+\beta)} \\
=\frac{(A-B)|\tau|}{E_{\alpha, \beta}(m)} \sum_{n=2}^{\infty} \frac{(1-\delta / n)}{\Gamma(\alpha(n-1)+\beta)} m^{n-1} \\
=\frac{(A-B)|\tau|}{E_{\alpha, \beta}(m)}\left[\sum_{n=2}^{\infty} \frac{m^{n-1}}{\Gamma(\alpha(n-1)+\beta)}-\sum_{n=2}^{\infty} \frac{\delta m^{n-1}}{n \Gamma(\alpha(n-1)+\beta)}\right] \\
=\frac{(A-B)|\tau|}{E_{\alpha, \beta}(m)}\left[\left\{E_{\alpha, \beta}(m)-\frac{1}{\Gamma(\beta)}\right\}-\delta\left\{\int_{0}^{m} \frac{E_{\alpha, \beta}(t)}{t} d t-\frac{1}{\Gamma(\beta)}\right\}\right] \leq 1-\delta .
\end{gathered}
$$

This completes the proof of theorem by given hypothesis.

Theorem 2.4. If $\alpha, m>0, \beta>1, f \in R^{\tau}(A, B)$ and the inequality

$$
\begin{aligned}
\frac{(A-B)|\tau|}{E_{\alpha, \beta}(m)} \frac{1}{\alpha}\left[\left(E_{\alpha, \beta-1}(m)-\frac{1}{\Gamma(\beta-1)}\right)\right. & \left.+\{\alpha(1-\delta)-(\beta-1)\}\left(E_{\alpha, \beta}(m)-\frac{1}{\Gamma(\beta)}\right)\right] \\
\leq & 1-\delta,
\end{aligned}
$$

is satisfied then $I(\alpha, \beta, m) f(z) \in K(\delta)$.

Proof. To prove $I(\alpha, \beta, m) f(z) \in K(\delta)$, we have to prove that

$$
\sum_{n=2}^{\infty} n(n-\delta) \frac{m^{n-1}}{\Gamma(\alpha(n-1)+\beta)} \frac{1}{E_{\alpha, \beta}(m)}\left|a_{n}\right| \leq 1-\delta .
$$

Now

$$
\begin{gathered}
\sum_{n=2}^{\infty} n(n-\delta) \frac{m^{n-1}}{\Gamma(\alpha(n-1)+\beta)} \frac{1}{E_{\alpha, \beta}(m)}\left|a_{n}\right| \\
\leq \frac{(A-B)|\tau|}{E_{\alpha, \beta}(m)} \sum_{n=2}^{\infty} \frac{(n-\delta) m^{n-1}}{\Gamma(\alpha(n-1)+\beta)} \\
=\frac{(A-B)|\tau|}{E_{\alpha, \beta}(m)} \sum_{n=2}^{\infty} \frac{(n-\delta) m^{n-1}}{\Gamma(\alpha(n-1)+\beta)} \\
=\frac{(A-B)|\tau|}{E_{\alpha, \beta}(m)} \sum_{n=2}^{\infty}(n-\delta) \frac{m^{n-1}}{\Gamma(\alpha(n-1)+\beta)}
\end{gathered}
$$




$$
\begin{gathered}
=\frac{(A-B)|\tau|}{E_{\alpha, \beta}(m)} \sum_{n=2}^{\infty} \frac{(n-\delta)}{(\alpha(n-1)+(\beta-1))} \frac{m^{n-1}}{\Gamma(\alpha(n-1)+\beta-1)} \\
=\frac{(A-B)|\tau|}{E_{\alpha, \beta}(m)}\left[\frac{1}{\alpha} \sum_{n=2}^{\infty} \frac{\left(n-1+1+\left(\frac{\beta-1}{\alpha}\right)-\left(\frac{\beta-1}{\alpha}\right)-\delta\right)}{\left\{(n-1)+\left(\frac{\beta-1}{\alpha}\right)\right\} \Gamma(\alpha(n-1)+\beta-1)} m^{n-1}\right. \\
=\frac{(A-B)|\tau|}{E_{\alpha, \beta}(m)} \frac{1}{\alpha} \sum_{n=2}^{\infty} \frac{\left[\left\{(n-1)+\left(\frac{\beta-1}{\alpha}\right)\right\}-\left(\frac{\beta-1}{\alpha}\right)+(1-\delta)\right]}{\left\{(n-1)+\left(\frac{\beta-1}{\alpha}\right)\right\} \Gamma(\alpha(n-1)+\beta-1)} m^{n-1} \\
=\frac{(A-B)|\tau|}{E_{\alpha, \beta}(m)} \frac{1}{\alpha} \sum_{n=2}^{\infty}\left[\frac{m^{(n-1)}}{\Gamma(\alpha(n-1)+\beta-1)}-\frac{(\beta-1) m^{n-1}}{\Gamma(\alpha(n-1)+\beta)}+\frac{\alpha(1-\delta) m^{n-1}}{\Gamma(\alpha(n-1)+\beta)}\right] \\
=\frac{(A-B)|\tau|}{E_{\alpha, \beta}(m)} \frac{1}{\alpha}\left[\left(E_{\alpha, \beta-1}(m)-\frac{1}{\Gamma(\beta-1)}\right)+\{\alpha(1-\delta)-(\beta-1)\}\left(E_{\alpha, \beta}(m)-\frac{1}{\Gamma(\beta)}\right)\right] \\
\leq 1-\delta .
\end{gathered}
$$

Thus the proof of Theorem 2.4 is established.

\section{REFERENCES}

[1] O.P. Ahuja, Connections between varioius subclasses of planar harmonic mappings involving hypergeometric functions, Appl. Math. Comput., 198 (1) (2008), 305-316.

[2] K.K. Dixit and S.K. Pal, On a class of univalent function related to complex order, Indian J. Pure Appl. Math., 26(9) (1995), 889-896.

[3] Manish Kumar, A study of univalent functions, M. Phil. Dissertation, C.S.J.M. University, Kanpur, India, 2014.

[4] G. Murugusundaramoorthy, K. Vijaya, and S. Porwal, Some inclusion results of certain subclass of analytic functions associated with Poisson distribution series, Hacettep J. Math. Stat., 45(4) (2016), 1101-1107, .

[5] S. Ponnusamy and F. Rønning, Starlike properties for convolutions involving hypergeometric series, Ann. Univ. Mariae Curie-Sklodawska SK Todawaska, 52 (1998), 141-155.

[6] Saurabh Porwal, An application of Poisson distribution series on certain analytic function, J. Complex Anal., 2014, Art. ID 984135 (2014), $1-3$, .

[7] Saurabh Porwal, Harmonic starlikeness and convexity of integral operators generated by generalized Bessel functions, Acta Math. Vietanm., 39 (3) (2014), 337-346.

[8] Saurabh Porwal, An application of certain convolution operator involving Poisson distribution series, Asian-European J. Math., 9(4) (2016), Art. ID 1650085, 1-8.

[9] Saurabh Porwal and Dilshad Ahmad, An application of hypergeometric distribution type series on certain analytic functions, Thai J. Math., Art. in press, (2019).

[10] Saurabh Porwal and Manish Kumar, A unified study on starlike and convex functions associated with Poisson distribution series, Afr. Mat., 27(5-6) (2016), 1021-1027.

[11] S. Porwal and Shivam Kumar, Confluent hypergeometric distribution and its applications on certain classes of univalent functions, Afr. Mat., 28 (2017), 1-8.

[12] S. Porwal and K.K. Dixit, On Mittag-Leffler type Poisson distribution, Afr. Mat., 28 (1-2) (2017), 29-34.

[13] M.S. Robertson, On the theory of univalent functions, Ann. Math., 37 (1936), 374-408.

[14] H. Silverman, Univalent functions with negative coefficients, Proc. Amer. Math. Soc., 51 (1975), 109-116, .

[15] A. Swaminathan, Certain sufficiency conditions on gaussian hypergeometric functions, J. Inequal. Pure Appl. Math., 5(4), (2004), Art. $83,1-10$. 